\title{
Methodology for Gas Industry Sustainable Development Strategy Based on the Ecological Imperatives
}

\author{
Alexander S. Kazak ${ }^{1, *}$ and Vladimir N. Bashkin ${ }^{2,3}$ \\ ${ }^{I}$ NIIGazeconomica LLC, Staraya Basmannaya st., 20-8, Moscow, 105066 Russia \\ ${ }^{2}$ Gazprom VNIIGAZ LLC, Razvilka, Leninsky District, Moscow Region, 142719 Russia \\ ${ }^{3}$ Institute of Physicochemical and Biological Problems of Soil Science RAS, Pushchino, Moscow Region 142292 Russia
}

\begin{abstract}
This paper is aimed to consider simultaneous development of gas production, processing and transportation capacities in the areas of the priority significance, such as in the Polar Regions of Russia. It was shown that the only way is creating a single strategy - master plan (scheme) of sustainable development of the gas industry based on the system approaches and ecological imperatives, at the local, regional and world scale.
\end{abstract}

Keywords: Gas industry chain, master plan, system approach.

\section{INTRODUCTION}

At present the global gas industry faces new challenges, both economical and environmental based. It is due to a significant gas production and consumption growth and major projects dedicated to both natural and liquid gas transportation systems creation from the new development and production areas, mainly in the remote areas, such as Polar Regions as the great example. Thus, simultaneous development of gas production, processing and transportation capacities have priority significance. Only creating a single strategy - master plan (scheme) of the sustainable development of the gas industry based on the system approaches and ecological imperatives could solve this task.

\section{CONCEPTUAL ISSUES}

In order to develop a methodology for designing a master scheme of gas industry sustainable development a comprehensive approach is needed to consider all areas of its activity (Kazak \& Bashkin 2007; Kazak \& Samsonov, 2007; Samsonov et al. 2007; Rusakova et al. 2009; Samsonov et al. 2007; Rusakova \& Kazak 2010), namely:

1) gas demand forecast;

2) gas export, import and transit;

3) forecast for development and reconstruction of natural gas production, transmission, underground storaging and processing facilities;

4) forecast for gas supply system development;

\footnotetext{
*Address correspondence to this author at the Institute of economics \& management of gas industry LLC, Gazprom (NIIgazeconomica), Staraya Basmannaya st., 20-8, Moscow 105066 Russia; Tel: +7 499 265-24-20; Fax: +7 499 267-30-76; E-mail: a.kazak@econom.gazprom.ru
}

5) assessment of the present status and development outlook of the mineral resource base of gas industry;

6) long-term evaluation of potential gas production;

7) assessment of reliability as well as industrial and environmental safety for gas facilities operations;

8) drawing up legal-regulatory framework for successful implementation of development projects.

Since these aspects are interconnected as a goal of this paper we will need systemic analysis methods applied to complex objects. In terms of systemic analysis, gas industry is a complex object that incorporates mineral resource base, gas production, processing, transmission, storage and consumption facilities. Each of these segments constitutes a complex subsystem (Kazak \& Bashkin 2007; Kazak \& Samsonov 2007).

\section{GAS SYSTEM AS A SUBJECT OF SYSTEM ANALY- SES}

With due regard to systemic analysis principles, gas industry may be represented as a complex graph with currently operating or potential gas transmission system (GTS) shown as its edges (Fig. 1).

The aggregation level of the graph depends on the set objectives. To formulate a country's gas industry development strategy, it is essential to design an upper-level scheme and to measure the balance between gas consumption and gas production (including gas import) (Kazak \& Samsonov 2007). Out of those nodes seen on Fig. (1), the first three refer to the input side of the balance (for the country under review) while the next three refer to the output side of the balance.

Gas storage systems may refer to both - the input side (gas extraction from storage facilities) and the output side of 


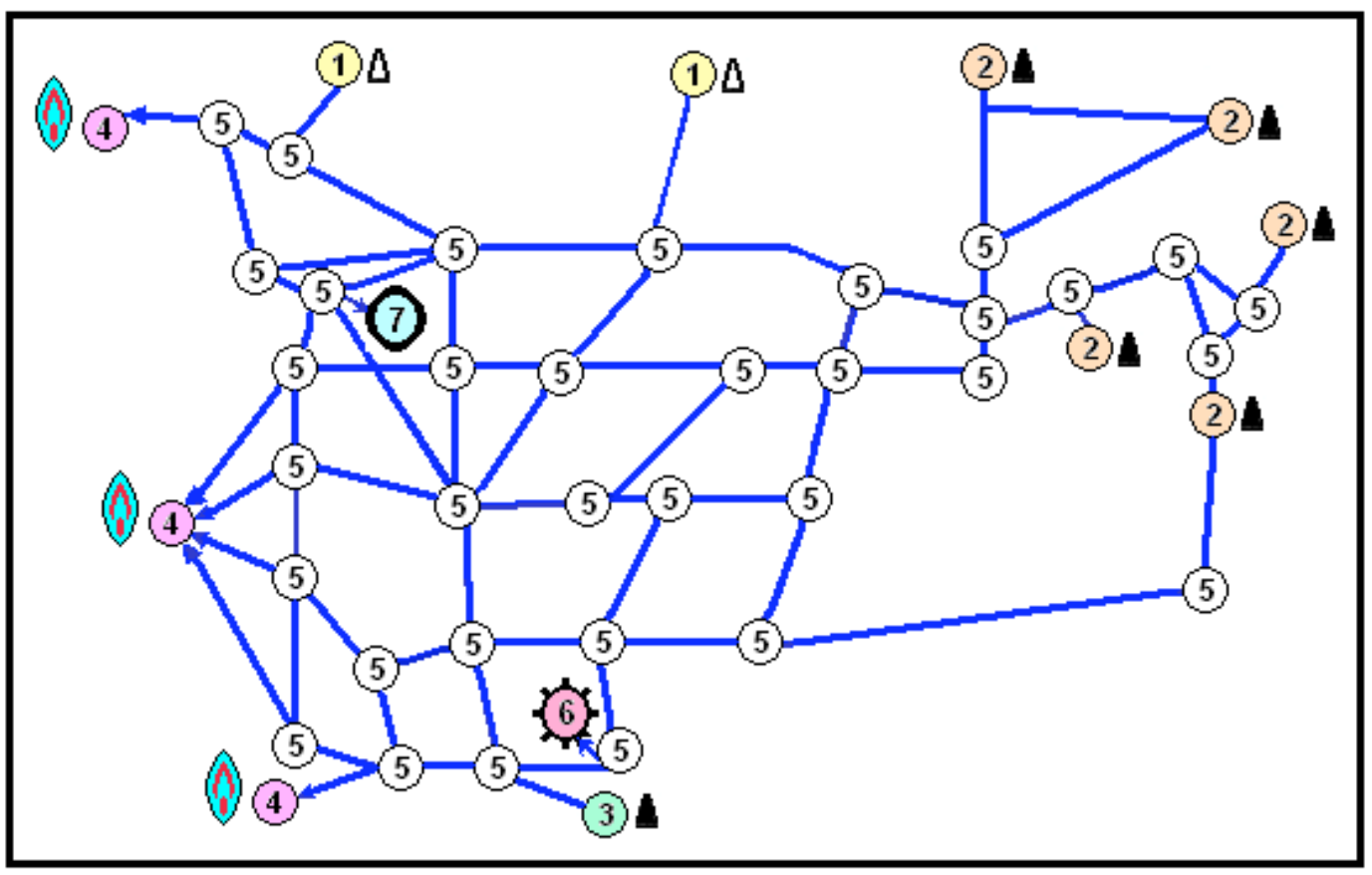

Fig. (1). Schematic representation of gas industry as a graph:

1 - potential gas-bearing provinces; 2 - existing gas-bearing provinces; 3 - gas suppliers from importing countries; 4 - export gas consumers; 5- internal gas consumers; 6 - gas processing facilities; 7- gas storage facilities.

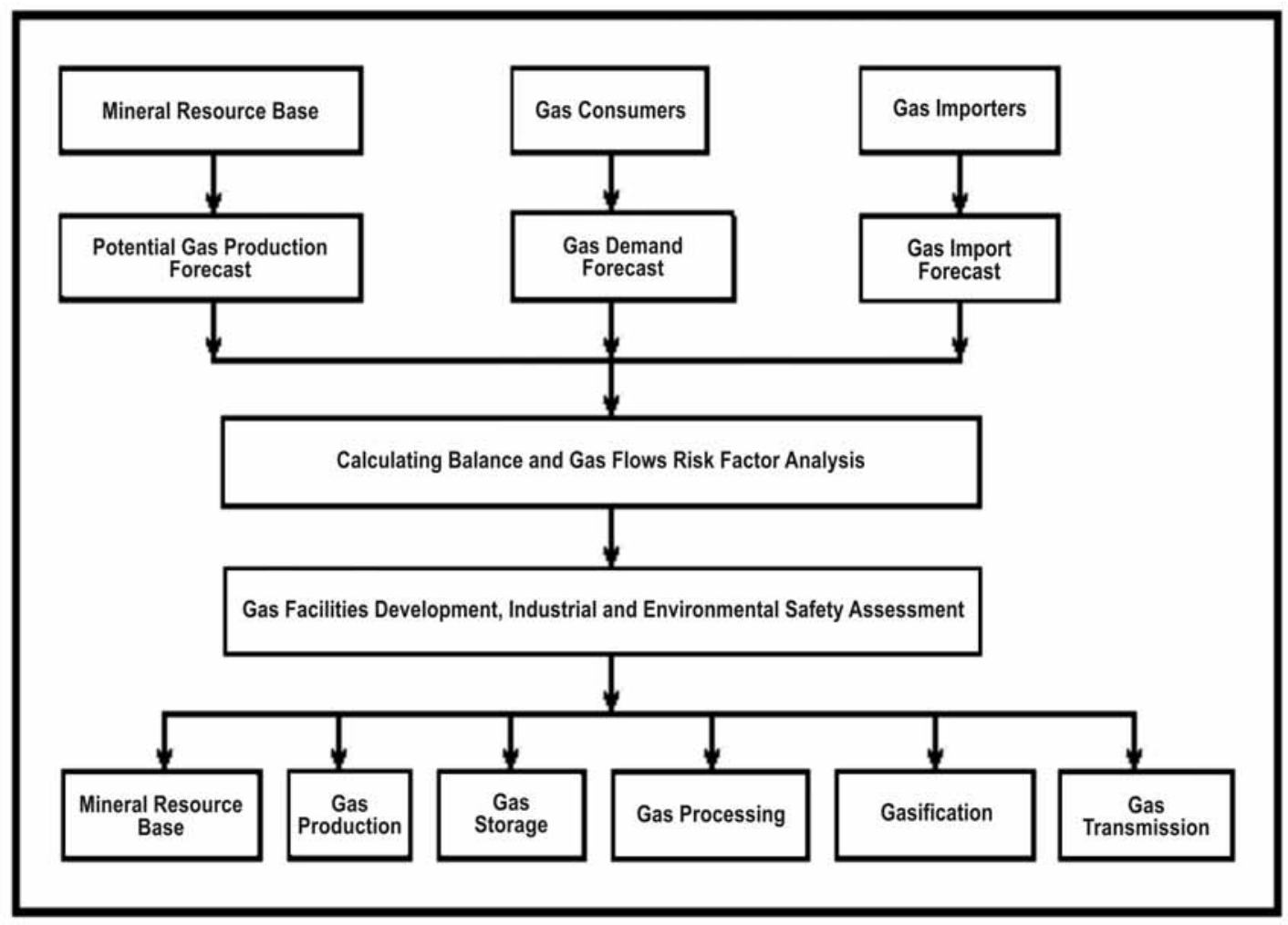

Fig. (2). Research block structure for gas industry development strategy.

the balance (gas pumping into storage facilities). Gas pipeline connectors are required to identify gas cross-flows between different pipeline corridors. In mathematical terms, the nodes referring to the input side of the balance have a positive potential, whereas those referring to the output side - a negative one.
Division of the whole gas industry system into segments as its separate subsystems allows to structure research block of gas industry development strategy at a lower level (Fig. 2).

Each unit in Fig. (2) constitutes a complex subsystem, which requires further decomposition down to the level 


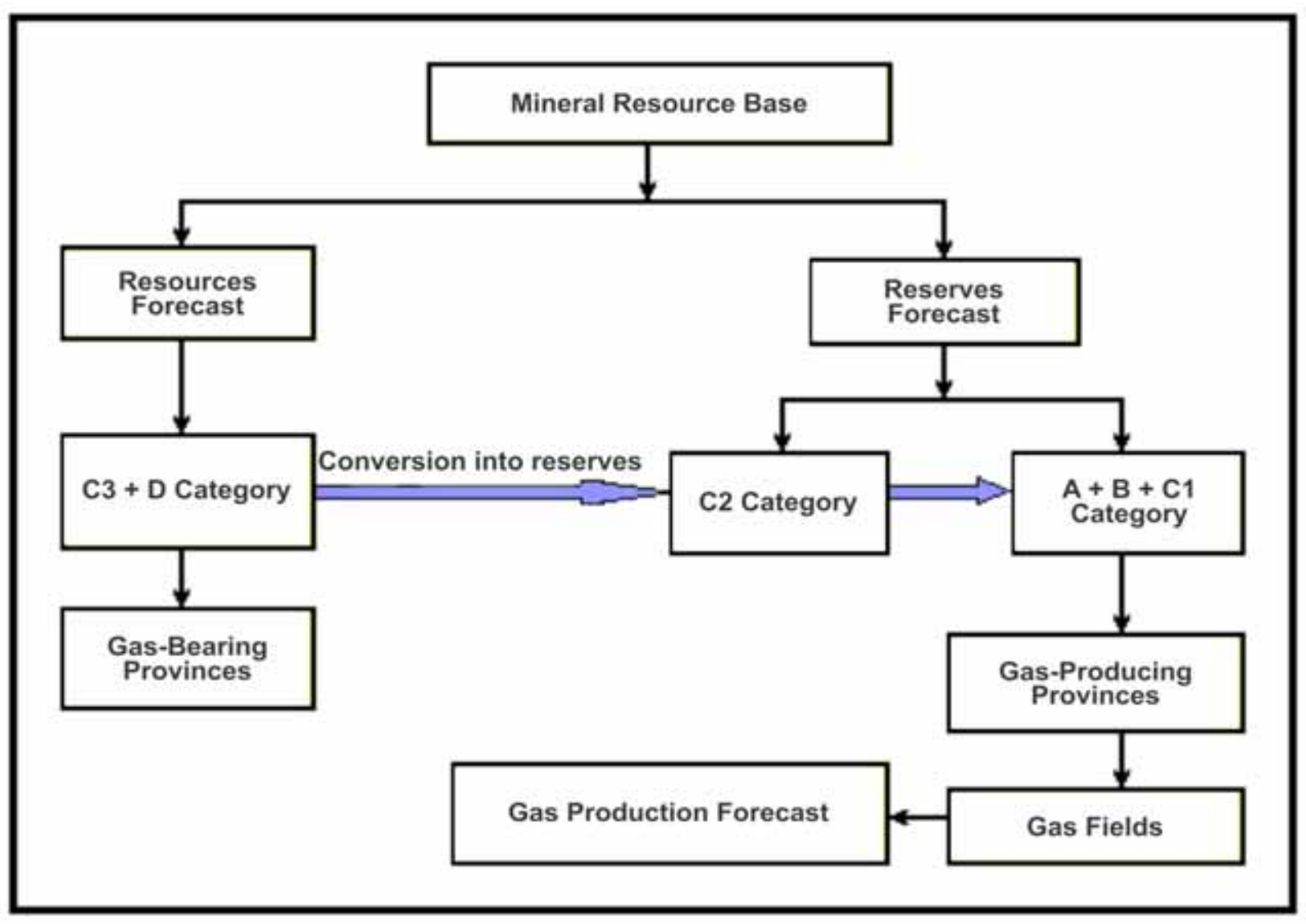

Fig. (3). Model of mineral resource base in gas industry: A, B, $C_{1}-$ developed gas reserves; $C_{2}-$ estimated gas reserves; $C_{3}-$ prospective gas resources; D - potential gas resources.

which enabled to describe each element via pertinent mathematical model.

A subsystem model of the mineral resource base includes forecast models of changes in gas resources and reserves (Fig. 3).

A forecast model for gas resource dynamics comprises pertinent models of gas resources categories, available in various gas-bearing provinces. To generate the prospective resources of the $\mathrm{C}_{3}+\mathrm{D}$ category, it is necessary to conduct 2D and 3D seismic surveys onshore and offshore and drill stratigraphic and appraisal wells. Apart from seismic surveys, electrical, gravity and magnetic exploration methods are applied on a smaller scale. In order to prove the resources and transfer them to the $C_{2}$ and $A+B+C_{1}$ reserve category, exploration drilling is performed with a subsequent interpretation of the data obtained in the course of geophysical well surveys. Such surveys involve application of the state-of-theart methods such as nuclear magnetic logging, electric, electromagnetic and acoustic scanning. As exploration wells are relatively widely spaced, prime importance is given to investigation of inter-well spaces by means of seismic, radioactive, acoustic logging and fluid dynamics analysis to convert the reserves to the $\mathrm{C}_{2}$ category. Depending on the geological survey results (including results of seismic surveys and exploration drilling) and their interpretation, a transfer is possible from the resource category to the $\mathrm{C}_{2}$ and
$\mathrm{A}+\mathrm{B}+\mathrm{C}_{1}$ reserves categories. As a result the total for proved gas reserves normally increases. However, the forecast of gas production dynamics may present lower numbers.

Changes forecast for the proved gas reserves and perspective resources allows to assess medium-term and longterm potentials of gas production for specific fields and gasbearing provinces. Thus, at the system synthesis stage, upper gas production limits in 1 and 2 aggregated nodes may be defined. (See Fig. 1). Moreover, while planning the development of gas production facilities, special attention should be given to technically feasible performance constraints in gas transmission system.

Accordingly, it is expedient to perform joint calculations to optimize gas production and transmission facilities development strategy. These calculations should result in forecasting gas production dynamics in the system components, as shown in Fig. (1). Such forecast may serve as the basis for calculating spread of similar forecast characteristics of gasbearing field groups within each node of the system. Calculations should be done at the stage of decomposing a gasbearing province into separate field groups. Moreover, according to the systemic analysis method, field groups should be further decomposed to specific gas fields and individual wells (Fig. 4).

To assess gas production facilities, a reservoir simulation model for field groups is applied. It allows to distribute 


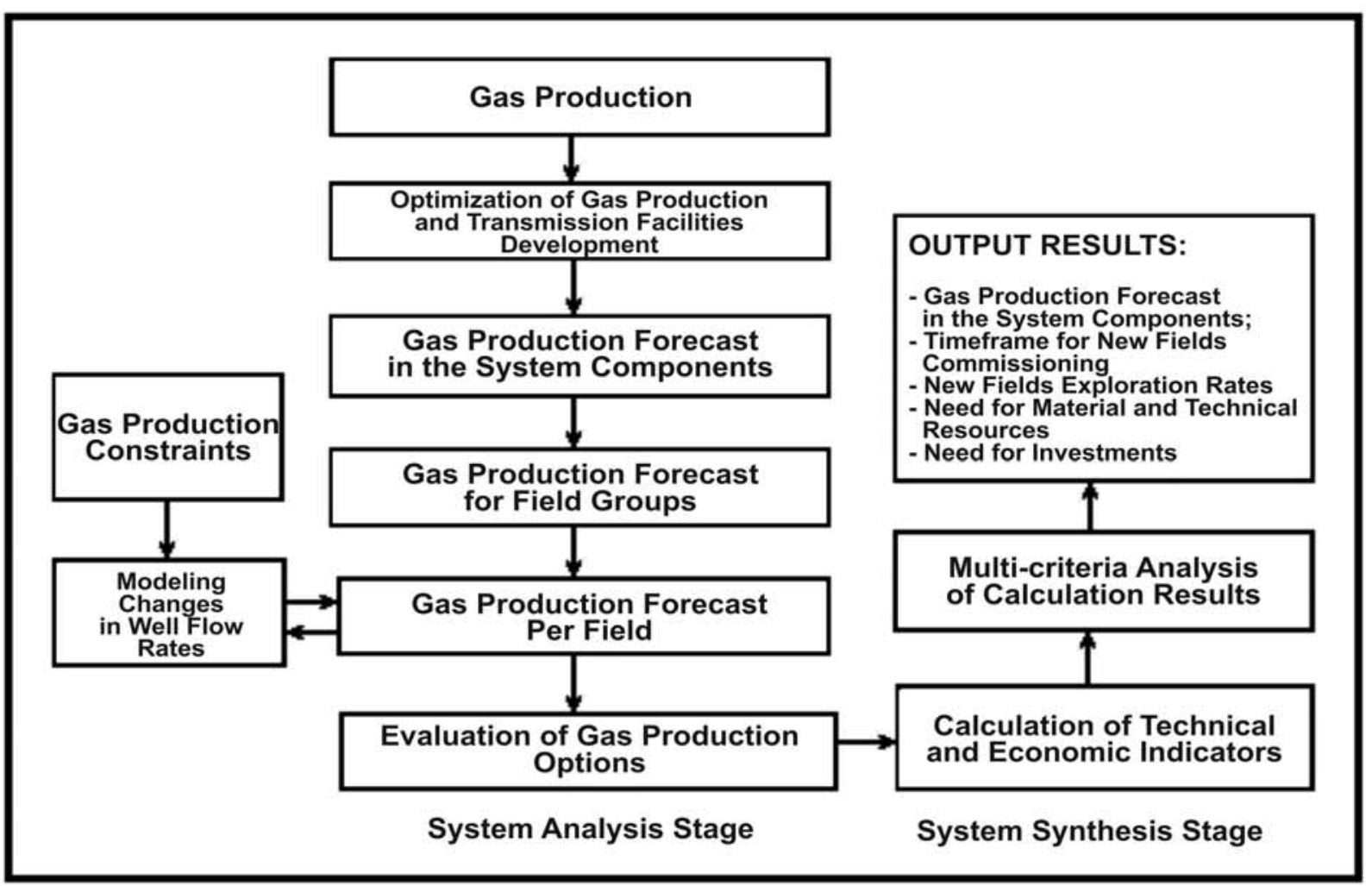

Fig. (4). - Forecast model of gas production dynamics.

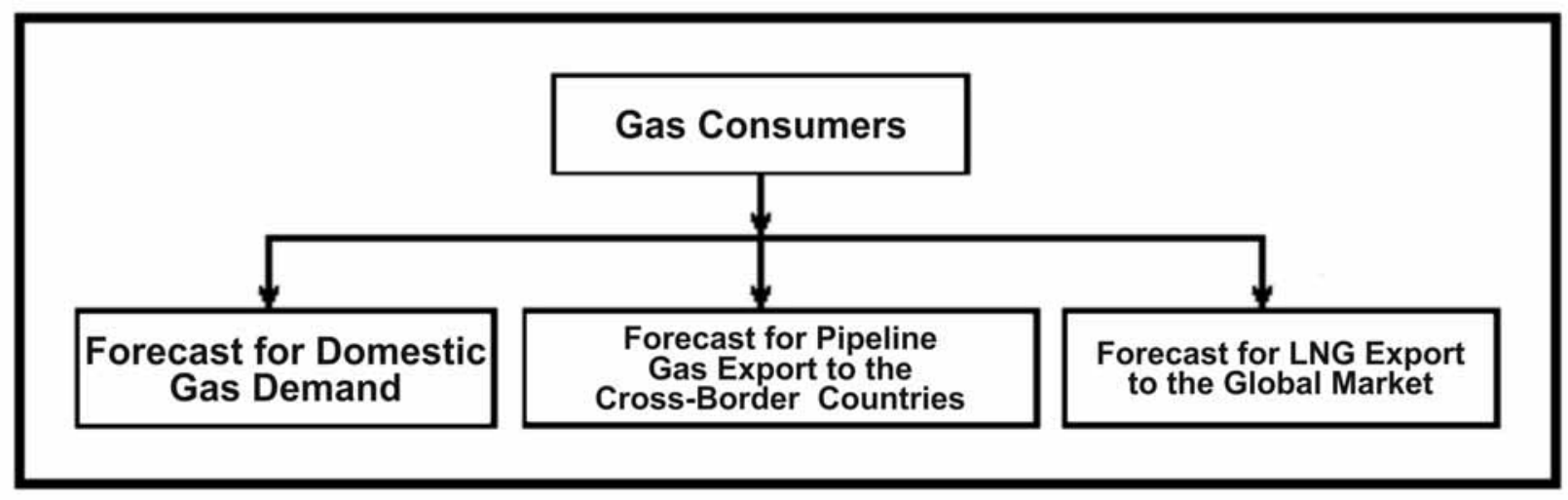

Fig. (5). Forecast model of gas demand: LNG - liquefied natural gas.

production volumes among the fields in a dynamic environment at the very start of calculation according to the input data on gas production forecast for field groups, gas reserves and fields plateau, and technical characteristics. A general reservoir simulation model of gas fields is structured as a system of differential equations with the corresponding initial and limitation conditions. Gas production parameter is measured as an integral characteristic of gas production dynamics per well, where the controlled parameter being a number of wells put into operation per unit of time. Setting different dynamic values for well drilling makes it possible to control changes in the development parameters for individual fields and field groups pertaining to the system components. Thus, different gas production options can be obtained, in consistency with gas production dynamics in the system components calculated at the stage of gas production and transmission optimization. Each option is developed with account of well start-up, operation and decommissioning phases.

After a set of options are calculated relying on multicriteria analysis and expert systems, the most acceptable one is selected. As a result, the priority order is established for each system component along with schedules for field startup, field development intensity and as well as the need for material and technical resources and investments.

A subsystem for modeling gas consumer demand includes forecast models of gas demand within a country, pipeline gas export to the cross-border states and natural liquefied gas, LNG, sales in the global energy market (Fig. 5). 
Such subsystem decomposition is tied up to the existing differences in gas prices in different countries and taxation structure. Mathematical models that involve evaluating the lower, medium and upper limits of the gas demand variation interval applying scientific approach for gas industry development strategy. In practice, consumers' demand is assessed according to gas consumption probability level (which is as a rule $90 \%, 50 \%$ and $10 \%$ respectively). As such, the lower gas demand level corresponds to the required gas volumes projected with a $90 \%$ probability, the medium demand level corresponds to a $50 \%$ probability, while the upper gas demand level corresponds to the required gas volumes with no less than $10 \%$ probability. Methodically this assessment includes various approaches to the scope of energy efficiency potential of the country's economy, availability of local projects aimed at developing gas consumers and prospects of funding. This is due to availability of different forecasts of the major macro- and microeconomic effect in shaping gas demand.

A scientifically substantiated forecast of gas exports appears to be an extremely difficult task as it requires an internal gas demand analysis in the potential gas-exporting countries, political and economic risks assessment, pipeline gas supply projects coordination between the cross-border countries and conclusion of pertinent long-term agreements. Methods of systemic analysis allow to decompose the forecast models along the export sections of operating and potential gas transmission pipelines.

Gas export forecast for a specified period of time should be based on assessment of gas demand development and determination of a potential market share for a country under review together with gas supply contracts drawn up and signed with the cross-border countries.

The forecast of LNG exports to global markets should be based on the prospective gas balance analysis in the countries acting as potential importers. It should also take into account information on the amounts needed to satisfy gas demand and expert evaluation. The world's growing demand for liquefied natural gas may serve as a good stimulus for a country to be a part of this process. This is strategically important since having missed the moment of market formation, the country will have to adapt to a newly shaped trade and competition system. Meanwhile, the world's top LNG importers are the countries in the Asia-Pacific Region.

Since gas import is a constituent part of the gas sector development in a vast number of countries, a mathematical model determining gas import dynamics must involve assessment of potential gas supplies from a country. It is normally based on estimation of potential gas production in various gas-bearing provinces of the exporting country, evaluation of domestic gas consumption, analysis of intergovernmental agreements and appraisal of the political situation. The last factor, however, may cause significant uncertainty of the forecast obtained. In this regard, it is advisable to estimate the minimum, maximum and the most likely gas import levels.

The forecast of the minimum import level should take into account risks associated with potential reduction in gas supplies from the importing countries due to the lack of sufficient resources, transmission of a portion of produced gas to other countries and political environment within a country. The medium import level may be estimated on the basis of the contractual gas volumes and potential gas production capabilities of the mineral resource base in the importing country. The maximum level depends on intergovernmental agreements, provided that new fields are developed in a country. Gas import and gas production forecasts at the synthesis stage form part of positive potential of the relevant system components (Fig. 1).

As a rule, there are fluctuations observed in gas supply of any country due to changes of gas supply and consumption volumes. Gas storage system is a tool that serves to enhance reliability and reduce fluctuations in gas supplies. It presupposes the construction of underground gas storage facilities in the areas close to major gas consumers, and creation of a network of natural gas liquefaction, storage and regasification facilities.

Decomposition of the gas storage subsystem (node 7, Fig. 1) involves consideration of various natural gas storage subsystems. Consequently, for programs of underground gas storage system development it is advisable to use mathematical models of gas underground storage facilities to calculate gas active volume. This in its turn would allow:

1 to overcome fluctuations in gas consumption;

2 to create long-term gas reservation facilities at the connecting points of gas transmission network and in proximity to strategically important gas consumers.

Furthermore, to relieve gas shortage at the peak of demand, it is practical to establish a network of liquefied natural gas storage facilities. Coupled with conversion to alternative fuels it could provide the required gas volumes.

Forecast for developing a specified gas industry subsystem results in determination of the construction period and technical specification of gas storage facilities along with demand for material and technical resources and investments.

Within the gas industry system, processing plants are referred to as consumers of natural gas. The natural gas processing subsystem presupposes the range of produced goods (liquefied hydrocarbon gas, methanol, motor fuels) and potential components which may be produced via deep conversion technologies (liquefied natural gas, polyolefins, synthetic liquid fuel, etc.). All the segments require special mathematical models that would ensure distribution in alignment with gas industry system components (see Fig. 1), construction period, demand for material and technical resources and investments.

Gas supply infrastructure like gas processing is a gasconsuming segment of gas industry. This subsystem is developed both on the national and local scales, with each country's sub-region having an opportunity to create its own gas supply development program as part of the national gas supply facilities development program. Its development technology also involves systems analysis methods, while the development strategy is similar to that of gas industry development. Meanwhile, it is worth noting that the dates and scope of construction set forth in the programs for gas supply plants construction should be consistent with development and reconstruction dates and scope for gas produc- 


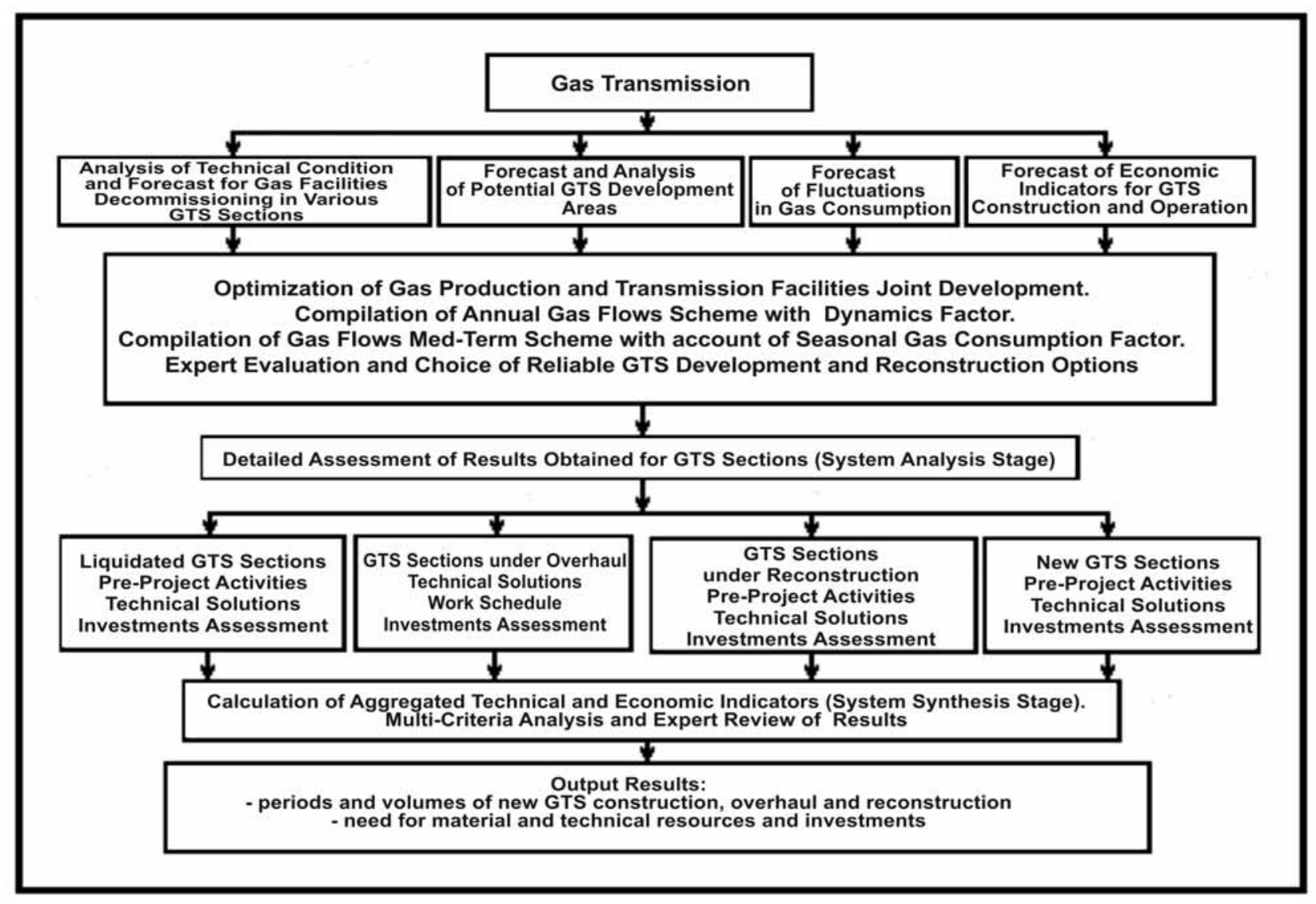

Fig. (6). - Forecast model for GTS reconstruction and development in the Unified Gas Supply System. GTS - Gas Transmission System.

tion facilities and trunk pipeline transmission system (development of local hydrocarbon resources and trunk pipelines construction). The strategy of regional gas supply infrastructure development requires creation of gas distribution networks thus ensuring supplies of compressed and liquefied hydrocarbon resources. The outcome of these programs should be gas demand forecast for the corresponding system components (see Fig. 1) and forecast of material and technical resources and investment demand.

The models of potential gas production assessment and forecasts of gas demand and import within the system components (including gas supply plants, gas processing and storage facilities), allow to calculate the overall gas balance. Since there are variations in the input and output sides of the balance, multiple calculations and diverse scenarios of gas industry development are required. In technical terms, the output side of the balance covers manufacturing and operational needs of the consumers, gas export and utilization in the cycling process, gas pumping into underground gas storage facilities (in the volumes required to overcome fluctuations in gas consumption), technological needs in the course of gas transmission, needs of gas industry, etc. The overall gas volumes required to ensure the output side of the balance may be generated by a combination of different sources. The current data on the input and output sides of the balance serve as the basis for creating gas balance options for further calculations. These options are comprised of gas balance for each individual country's province or sub-regions connected by gas transmission networks. Forecast of development dynamics for gas facilities and the whole system is made relevant to each balance option. As a result, a set of alternative options of gas industry development are put forth.

To adequately assess whether further calculations are needed with respect to different options, risk factor forecast and analysis are required. Potential change in gas demand is attributed to risk factors as well as lack of tested technologies of field development in new areas, a potential decline in gas imports, restrictions related to environmental and industrial safety, etc. A preliminary analysis of risk factors enables to cut off the most risky options of gas industry development strategy at an early stage.

Gas transmission system (GTS) plays a special role in gas industry of any country, since it transmits gas flows from one system component to the other. In Fig. (1) all the constituent parts of gas industry are represented as nodes and the gas transmission system is shown as links between them. Gas transmission is the most capital-intensive sector of gas industry, accounting for up to $85 \%$ of fixed assets value in different countries. Development of new gas-bearing provinces should inevitably lead to significant modifications in the gas flows scheme and require enormous financial investments. Forecasts of development and reconstruction scope for the gas transmission system (as well as other gas facilities) are made in two stages, with systemic tasks fulfilled at the first stage and technical solutions for individual GTS sections developed at the second stage (Fig. 6). Calculations are made in parallel with gas facilities and gas 
Table 1. Assessment algorithms for optimization of gas industry sustainable development.

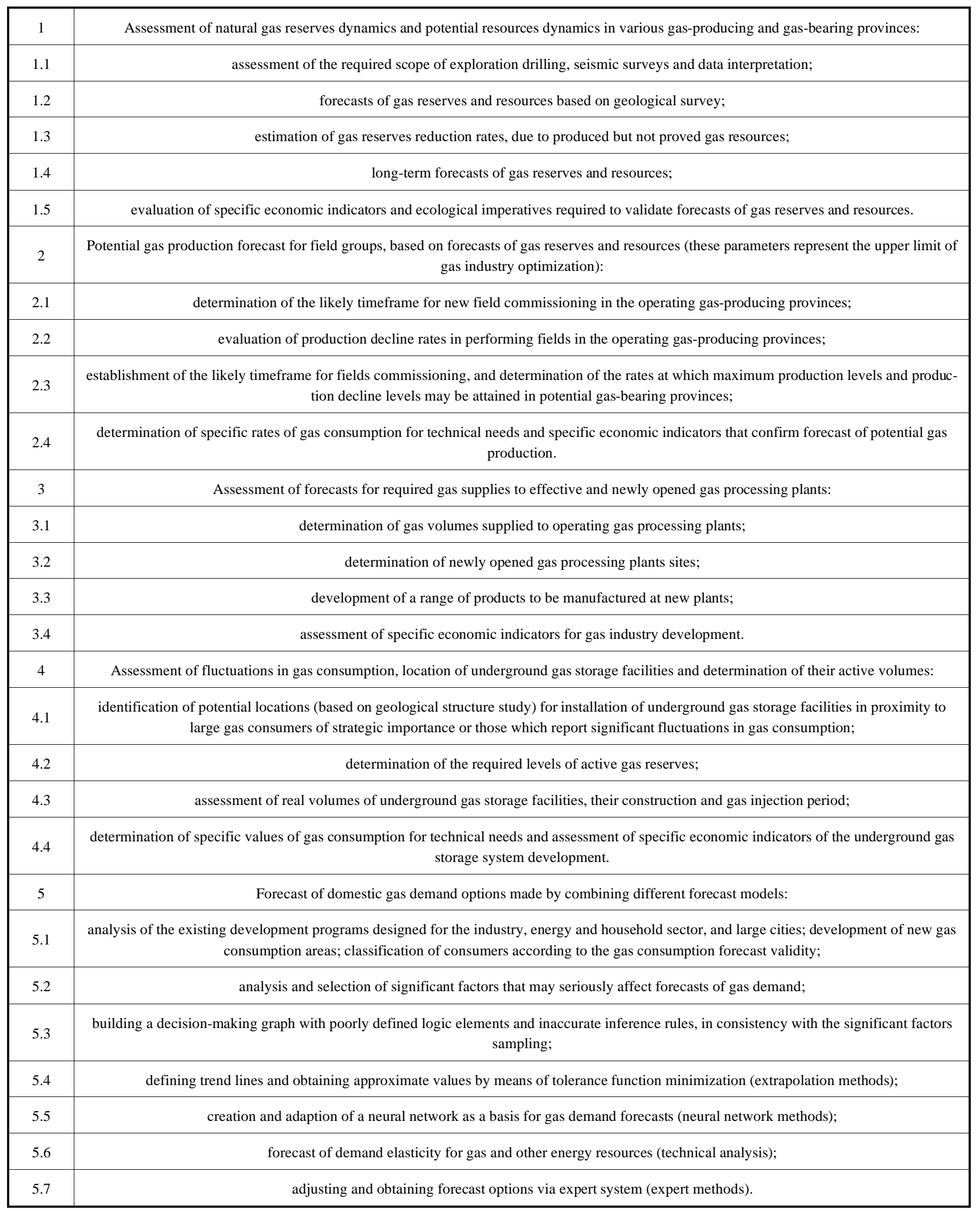


Table 1. contd...

\begin{tabular}{|c|c|}
\hline 6 & Assessment of potential gas import and export levels: \\
\hline 6.1 & $\begin{array}{l}\text { determination of the required gas export and import volumes as a difference between potential gas production and domestic gas demand options } \\
\text { (export in case of positive difference, import in case of negative difference); }\end{array}$ \\
\hline 6.2 & identification of potential gas importing countries; forming nodes for imported gas inlet into the gas transmission system; \\
\hline 6.3 & assessment of possible imported gas supply levels (lower, medium and upper); \\
\hline 6.4 & assessment of specific economic indicators for each gas import level; \\
\hline 6.5 & $\begin{array}{l}\text { identification of potential export gas consuming countries; forming nodes for pipeline gas outlet from the gas transmission system and evalua- } \\
\text { tion of export gas supply forecasts; }\end{array}$ \\
\hline 6.6 & assessment of specific economic indicators of pipeline gas export supplies; \\
\hline 6.7 & $\begin{array}{l}\text { identification of potential for liquefied natural gas consuming countries; forming nodes for gas transfer from the gas transmission system to } \\
\text { liquefied natural gas production plants, and evaluation of the export supply forecasts; }\end{array}$ \\
\hline 6.8 & assessment of specific economic indicators of liquefied natural gas export supplies. \\
\hline 7 & Synthesis of gasification facilities and assessment of the total gas consumption in GTS components: \\
\hline 7.1 & creating GTS components linked to gasification facilities; \\
\hline 7.2 & assessment of gas potential consumption in GTS components on the basis of available gas demand forecast; \\
\hline 7.3 & assessment of specific economic indicators relating to development of particular gasification facilities; \\
\hline 7.4 & assessment of specific economic indicators of the gas supply system development with regard to particular GTS components. \\
\hline 8 & Joint optimization of gas industry development and gas facilities reconstruction aided by interactive export system: \\
\hline 8.1 & $\begin{array}{c}\text { development of gas transmission system topology (with the use of geo-information systems) by adding, removing and creating reversible curves, } \\
\text { adjusting new components of gas consumption and components of gas utilization for GTS technical needs; determination of distances between } \\
\text { the GTS components; }\end{array}$ \\
\hline 8.2 & $\begin{array}{l}\text { evaluation of gas pipeline technical condition; forecast for decommissioning of capacities and technically feasible performance of GTS sections; } \\
\text { identification of restrictions for the curves throughput capacity; }\end{array}$ \\
\hline 8.3 & $\begin{array}{l}\text { assessment of specific weight values of network curves (using monetary terms), based on specific economic indicators (with application of more } \\
\text { precise economic indicators where project or pre-project findings are available); }\end{array}$ \\
\hline 8.4 & $\begin{array}{l}\text { determination of specific values of gas consumption for pipeline technical needs; development of gas balance options and assessment of specific } \\
\text { weight values (using monetary terms) of network components, based on specific economic indicators (with application of more precise eco- } \\
\text { nomic indicators where projects or pre-project findings are available); }\end{array}$ \\
\hline 8.5 & $\begin{array}{l}\text { optimization of gas flows (iterative, starting with Item } 8.3 \text { ) in consistency with reconstruction and development of gas production, transmission, } \\
\text { and storage facilities, with application of a long-term income maximization criterion; }\end{array}$ \\
\hline 8.6 & $\begin{array}{l}\text { selection of appropriate options (by a group of experts) for gas industry reconstruction and development, based on expert system evaluation } \\
\text { (some options may be rejected in case of non-compliance with laws, legal acts, applicable norms, industrial and environmental safety standards, } \\
\text { high risks associated with projects implementation, including financial risks, non-performance of the state social and economic programs). }\end{array}$ \\
\hline 9 & Detailed assessment of calculation results obtained for gas facilities: \\
\hline 9.1 & $\begin{array}{l}\text { decomposition of each component representing a gas production facility into separate elements (fields, wells, boosters, field facilities, inter-field } \\
\text { pipelines, etc.); expert system facilitates selection of components subject to decommissioning, conservation, overhaul, reconstruction and new } \\
\text { construction; running pre-project activities; development of process solutions and making detailed evaluation of economic indicators; }\end{array}$ \\
\hline
\end{tabular}


Table 1. contd...

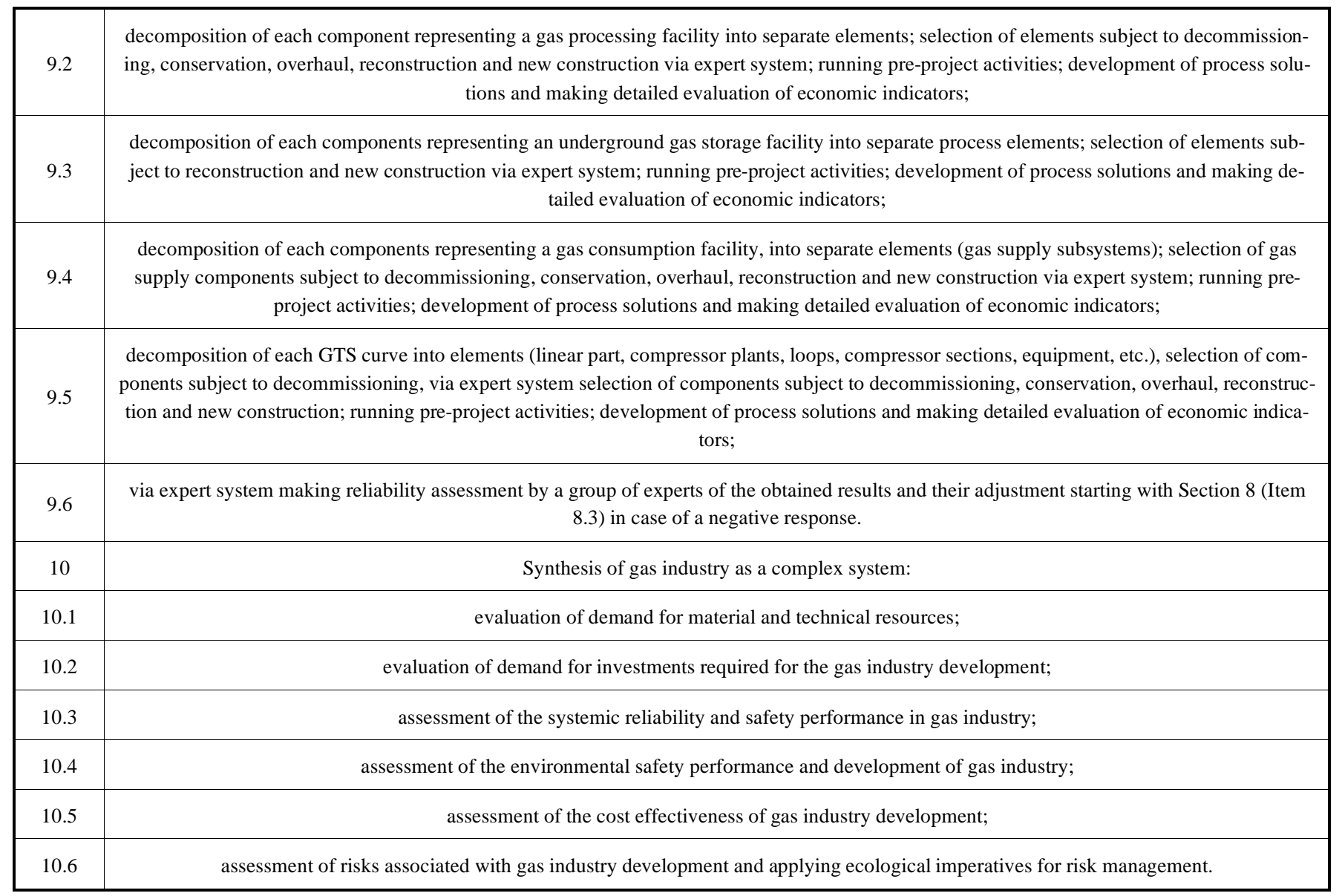

balance development options. A preliminary analysis of technical condition and forecast for potential gas facilities decommissioning in various GTS sections are made, in case of a wait-and-see approach, without overhaul and reconstruction. Forecasts of the timeline for new gas-bearing provinces exploration are used to identify potential GTS development areas. The preliminary assessment stage involves forecasts of gas consumption fluctuations and economic indicators for GTS construction and operation. This stage also involves multi-criteria optimization of the annual gas flows scheme. As a final evaluation criterion, economic indicators may be used (e.g., maximum discounted net revenue). This approach transforms a problem into a non-linear one and, therefore, requires iterative recalculation of the criterion per se and the optimum gas flows.

Presumably, a minimum level of commodity transportation activity may serve as an optimization criterion. However, gas flows scheme optimization is performed for the entire gas industry development period, which makes it necessary to solve a non-linear dynamic transmission problem. Thus, special algorithms are proposed for optimization of gas industry development (Table 1) (Kazak et al. 2007a; Kazak et al. 2007b; Kazak et al. 2007c; Kazak et al. 2007d; Kazak et al. 2008).

\section{OPTIMIZATION OF GAS INDUSTRY SUSTAINABLE DEVELOPMENT}

Models and methods applied at the first stage allow to assess production rates in GTS sections. As a result, a set of options for GTS development and reconstruction are established and assessed through specially developed expert system.

Thus, the second stage involves assessment of development and reconstruction scope in the gas transmission sector. An expert system is applied to select gas pipeline sections subject to decommissioning, conservation, overhaul, reconstruction and new construction. To carry out pre-project activities, develop process solutions and estimate investments each GTS section is decomposed into separate elements, which cover a linear part, compressor plants, loops, compressor sections, equipment, etc. The obtained results allow to assess the level of industrial safety and the degree of geoecological risks for various gas pipeline components.

The stage of GTS synthesis requires return to its integrated scheme and evaluation of the consequences of potential emergency situations in order to determine the system's compensatory capabilities and gas supply reliability. As a result of gas volumes optimization required for the transmission subsystem a new gas supply scheme is devised. Priory 
order and timing of gas pipeline construction and reconstruction are determined and the need for material and technical resources and investments is evaluated.

Such an approach is the only manageable in the remote areas of modern and prospective gas production - in the global polar areas. Moreover, it will require the assessment of risks associated with gas industry development and applying ecological imperatives for risk management.

\section{CONCLUSION}

Thus one may conclude that the only way of gas industry sustainable development is the creating a single strategy master plan (scheme) based on the system approaches and ecological imperatives, especially at the local and regional areas such as in the Polar Regions of Russia.

\section{CONFLICT OF INTEREST}

The authors confirm that this article content has no conflicts of interest.

\section{ACKNOWLEDGEMENTS}

The authors express their deep appreciation and gratitude for their affiliation institutions, namely Institute of economics and management of gas industry, NIIGazecomonica, and
Institute of natural gases and gas technologies, Gazprom VNIIGAZ, for the support during research and development.

\section{REFERENCES}

Kazak \&AS, Bashkin, VN (2007) Advanced principles of gts development and reconstruction scheme for the unified gas supply system. Management Systems and Information Technologies, 2.1 (28), 144-8.

Kazak, AS \& Samsonov, RO (2007) Systems analysis for geoenvironmental risks minimization in complex trunk pipelines. Management Systems and Information Technologies, 3.1 (29), 1426.

Kazak, AS, Kislenko, NA, Kazak, KA \& Bashkin, VN (2007a) Expert assessment in gas demand forecast. Oil, Gas and Business, 10, 67-72.

Kazak, KA, Knot'ko, VV, Kislenko, NA \& Kazak, AS (2007b) Partial smoothing method in dispatch data correction. Management Systems and Information Technologies, 4.1 (30), 152-5.

Kazak, KA, Knot'ko, VV, Kislenko, NA \& Kazak, AS (2007c) Process data regression analysis. Management Systems and Information Technologies, 4.1 (30), 155-9.

Kazak, KA, Knot'ko, VV, Kislenko, NA \& Kazak, AS (2007d) Autoregressive model in forecast accuracy improvement. Management Systems and Information Technologies, 4.1 (30), 247-9.

Kazak, KA, Knot'ko, VV, Kislenko, NA \& Kazak, AS (2008) Neuron network forecast of GTS parameters. Management Systems and Information Technologies, 1 (31), 56-8.

Rusakova, VV, Kazak, AS \& Samsonov, RO (2009) Master Plan Methodology for Gas Industry Development, Gazprom VNIIGAZ, Moscow.

Rusakova, VV \& Kazak, AS (2010) Systems analysis in methodology for gas industry development. Science and Technology in Gas Industry, 2(42), 5-14.

Samsonov, RO, Kazak, AS \& Bashkin, VN (2007) Master Plan Methodology for Gas Industry Development, Scientific World, Moscow.

Samsonov, RO, Kazak, AS, Bashkin, VN, \& Lesnykh, VV (2007) Systems Analysis of Geo-Environmental Risks in Gas Industry, Scientific World, Moscow. 